\title{
POLPA REFINADA DE MAÇÃ PROMOVE ARRASTE VIA FECAL DE NUTRIENTES INGERIDOS NA DIETA
}

\author{
DRAGGING IN FAECES OF NUTRIENT INTAKE \\ TOGETHERWITH REFINED APPLE POMACE
}

\author{
Dorivaldo da Silva Raupp ${ }^{1}$, Ana Candida Vivan Cremasco², \\ Carla Marielle Caldi', Silvia Helena de Paula Marques², \\ David Ariovaldo Banzatto ${ }^{3}$
}

1 Autor para contato: Universidade Estadual de Ponta Grossa - UEPG, Campus em Uvaranas, Departamento de Engenharia de Alimentos, Ponta Grossa, PR, Brasil; (42) 220-3083; e-mail: raupp@uepg.br

2 Universidade Estadual de Ponta Grossa - UEPG Campus em Uvaranas, Laboratório de Tecnologia de Alimentos (F-29), Ponta Grossa, PR

3 UNESP, FCAV - Departamento de Ciências Exatas, Campus de Jaboticabal, Jaboticabal, SP

Recebido para publicação em 16/12/2004

Aceito para publicação em 04/05/2005

\begin{abstract}
RESUMO
Produtos farináceos produzidos a partir de subprodutos de agroindústrias alimentícias, como o bagaço de maçã, foram avaliados em pesquisas anteriores, as quais sugeriram o aproveitamento como alternativa de fonte de fibra alimentar para a alimentação humana. A pesquisa atual teve por proposta determinar o arraste, nas fezes, de nutrientes ingeridos na dieta juntamente com a polpa refinada de maçã (PRM) ou com o padrão farelo de trigo (FT), em ratos. Um dos dois nutrientes, a PRM ou o FT, foi incorporado à ração dos ratos na proporção de $5 \%, 15 \%$ ou $25 \%$, substituindo igual quantidade de amido. Ratos que receberam as fontes de fibra PRM ou a FT produziram, para todos os níveis testados na dieta, arrastes a partir da ingestão para as fezes semelhantes para o total de matéria sólida, de minerais, de proteínas, de lipídeos e de fibra alimentar associada aos carboidratos digeríveis. A fibra alimentar da fonte PRM foi a mais degradada pela biota microbiana do trato digestivo do rato, apresentando grau de hidrólise estimado em $59,04 \%$, enquanto que o do FT foi de $26,82 \%$.
\end{abstract}

Palavras-chave: farelo de trigo, fibra alimentar, alimento funcional, nutrição.

\author{
ABSTRACT \\ In a previous research, by-products such as apple pomace, generated by


food industries, were evaluated and indicated as an alternative source of dietary fiber for human nutrition. This study aimed to determine the dragging by faeces of nutrients taken in by young rats, together with refined apple pomace (RAP) or standard wheat bran (WB). RAP or WB, in the amounts of $5 \%, 15 \%$ or $25 \%$, were fed to the rats in substitution for starch and produced dragging proportions in the faeces equivalent to the total intake of dry matter, minerals, proteins, lipids, dietary fiber and digestible carbohydrates. The dietary fiber RAP presented the highest degree of degrading by the rats' digestive biota and it also presented the highest degree of hydrolysis, $59.04 \%$, while WB only presented a $26.82 \%$ hydrolysis degree.

Key words: wheat bran, dietary fiber, functional food, human nutrition

\section{Introdução}

A industrialização de maçã para a obtenção de suco, sidra e geléia produz quantidades apreciáveis de descarte sólido (bagaço ou polpa refinada) o qual continua sendo pouco aproveitado no sentido de "agregar valor” à produção. O uso dessa matéria alimentícia para rações de animais tem sido uma alternativa pouco rentável e, por vezes, até insignificante. A agro-indústria de maçã tem mostrado interesse em alternativas econômicas e tecnologicamente viáveis para a utilização desse descarte sólido e aguarda sugestões para que esse produto deixe de ser considerado, embora quantitativamente relevante, apenas como resíduo. Assim, visando aproveitar, para fins alimentícios, a matéria sólida produzida como descarte nas unidades de agroindústria de maçã, pesquisadores brasileiros e da comunidade científica mundial (Walter et al., 1985; Chen et al., 1988ab; Wang e Thomas, 1989; Renard e Thibault, 1991; Carson et al., 1994; Protzek et al., 1998; Raupp et al., 2000) avaliaram seus constituintes químicos bem como suas propriedades fisiológicas e nutricionais em experimentação com animais. A sua incorporação como ingrediente na formulação de alimento destinado à dieta humana foi avaliada por algumas dessas pesquisas.

A importância da fibra alimentar para a dieta humana está amplamente documentada na literatura específica e foi primeiro indicada como reguladora das funções digestivas. Posteriormente, foram atribuídos às fibras vários benefícios para a saúde humana, como a prevenção e o tratamento da diverticulite, hiperlipide- mia, hiperglicemia, câncer do intestino grosso (Smith et al., 1981; Eastwood et al., 1984; Schneeman, 1987; Roehrig, 1988; Seva-Pereira et al., 1991; Schweizer e Edwards, 1992). Perda de peso corporal em humanos foi observada em 1984 por Krotkiewski em indivíduos alimentados com goma guar. O mesmo foi observado por Davies et al., em 1991, os quais forneceram a ratos, dietas contendo fibras purificadas de pectina e de celulose. Redução da ingestão de alimentos e redução do ganho de peso corporal foram observadas em 1994 por Áreas, em ratos em dieta contendo polpa de laranja, a qual continha $71,1 \%$ de fibra detergente neutra que era constituída de $25,7 \%$ de celulose, 2,1\% de hemicelulose, 4,4\% de lignina e 38,9\% de substâncias pécticas.

Algumas fibras apresentam a capacidade de complexar-se com outros constituintes da dieta, como as substâncias tóxicas, podendo arrastá-los para a excreção fecal, dependendo do tipo de fibra. Os nutrientes da dieta, como proteínas, minerais, carboidratos digeríveis e lipídeos, também poderão ser excretados em maior ou menor quantidade, dependendo da fibra presente na dieta (Walker, 1975; Toma e Curtis, 1986; Davies et al., 1991; Seva-Pereira et al., 1991; Schweizer e Edwards, 1992; Raupp e Sgarbieri, 1996; Raupp et al., 2000,2002).

Proposta de estudo com a polpa de maçã, uma sobra do processamento de suco em laboratório, foi desenvolvida por Raupp et al. (2000), tendo por fim acrescentar mais informações e comparando-a ao farelo de trigo, em especial com relação às suas propriedades nutricionais e funcionais digestivas. O produto farináceo 
preparado por Raupp et al. (2000), chamado de polpa refinada de maçã (PRM), apresentou (Tabela 1) 91,91\% do seu peso seco em fibra alimentar, enquanto que o padrão farelo de trigo (FT) apresentou 43,69\%. E os conteúdos de carboidrato digerível, proteína, lipídeo e cinza foram, respectivamente, iguais a $0,00 \%$
- 8,20\% - 0,57\% -2,04\% para a PRM e 27,92\% $19,79 \%$ - 3,43\% - 6,33\% para o FT. Portanto, a fibra alimentar representou quase que a totalidade do peso seco da PRM, destacando esse produto farináceo como uma fonte contendo elevado conteúdo de fibra alimentar, 2,1 vezes mais fibra que o FT.

Tabela 1 - Composição nutricional (\%, base seca) ${ }^{1}$ da polpa refinada de maçã (PRM) e do farelo de trigo (FT), utilizadas na formulação de dietas para os ratos.

\begin{tabular}{|c|c|c|c|c|c|}
\hline Fonte de fibra & $\begin{array}{c}\text { Fibra } \\
\text { alimentar }\end{array}$ & $\begin{array}{c}\text { Carboidrato } \\
\text { digerível }^{4}\end{array}$ & Proteína & Lipídio & Cinza \\
\hline $\mathbf{P R M}^{2}$ & $91,91^{5}$ & 0,00 & 8,20 & 0,57 & 2,04 \\
\hline $\mathbf{F} \mathbf{T}^{3}$ & 43,69 & 27,92 & 19,79 & 3,43 & 6,33 \\
\hline
\end{tabular}

O objetivo deste trabalho foi avaliar a excreção fecal de nutrientes em ratos que receberam rações de polpa refinada de maçã em concentrações de 5\%, 15\% ou $25 \%$ e tendo como padrão de fibra alimentar o farelo de trigo obtido do comércio.

\section{Material e métodos}

A fonte de fibra alimentar "polpa refinada de maçã (PRM)" foi produzida em laboratório, conforme protocolo descrito por Raupp et al. (2000). A PRM é a matéria sólida que sobra depois de extraído o suco na presença de enzimas pectinolíticas, seguida de lavagem com água, secagem ao sol e trituração em processador de alimentos. O farelo de trigo (FT), fonte padrão de fibra usada para comparação, foi obtido do comércio. As composições nutricionais da PRM e do FT estão apresentadas na Tabela 1.

O produto obtido PRM foi mantido em embalagem hermética até a sua utilização nos ensaios biológicos, os quais foram realizados com ratos modelo, em crescimento, e tendo como padrão de fibra o farelo de trigo (FT) adquirido no comércio. O ensaio biológico consistiu de sete tratamentos, cada um com seis ratos, distribuídos entre os tratamentos segundo o modelo de blocos, ao acaso. Ratos de três tratamentos receberam a PRM nas concentrações de 5\%, 15\% e 25\%; ratos de três tratamentos receberam o FT como padrão, nas concentrações de 5\%, $15 \%$ e $25 \%$; e um grupo (tratamento) de ratos não recebeu fonte de fibra. No preparo das rações foram utilizados os seguintes ingredientes: caseína comercial fornecida pela Kauffmann \& Co., contendo 90,39\% de proteína (\%N x 6,40), em base seca; óleo refinado de soja; amido de milho fornecido pelo produto comercial "Maizena", de grau de pureza igual a 99,8\%, em base seca; mistura vitamínica e mistura de sais minerais, preparadas em laboratório segundo a formulação da AIN-93G (Reeves et al., 1993). A ração base (sem fonte de fibra) foi preparada segundo a formulação da AIN-93G (Reeves et al., 1993): 20\% de caseína; $7 \%$ de óleo de soja; 3,5\% de mistura mineral; $1 \%$ de mistura vitamínica; 0,3\% de L-cistina; 0,25\% de bitartarato de colina. O amido foi a única fonte de carboidrato digerível e entrou na dieta base em q.s.p. $100 \%$. Um dos dois nutrientes - a PRM ou o FT entrou na formulação das dietas como fonte de fibra alimentar para os ratos e substituindo igual porcentagem de amido, de $5 \%, 15 \%$ ou $25 \%$. A 
formulação da AIN-93G (Reeves et al., 1993) considera $5 \%$ o teor de fibra alimentar para a dieta normal de ratos.

Os animais modelo utilizados foram ratos albinos da linhagem Wistar, SPF (Livres de Patógenos Específicos), de 21 dias e fornecidos pelo Biotério Central da Universidade Federal de Santa Catarina (UFSC). A temperatura do Laboratório de Ensaios Biológicos foi mantida a $21 \pm 2^{\circ} \mathrm{C}$, com alternância automática de claro-escuro em períodos de 12 horas. Os animais receberam dieta e água ad libitum. O ensaio teve duração de 33 dias, incluindo 5 dias de adaptação ao ambiente de experimentação e 28 dias para coleta de dados.

Durante o ensaio foram feitas, de cada rato, as determinações de peso de alimento ingerido e de peso seco das fezes coletadas. Nas fezes do rato foi determinada a umidade, o total de minerais (como cinzas), a proteína (\%N x 6,40) e os lipídeos, segundo os métodos publicados na Association of Official Analytical Chemists (1984). A fração de carboidratos, que incluiu a fibra alimentar, foi determinada nas fezes por diferença porcentual. A proporção do total da ingestão excretada como fezes foi calculada levando em conta os pesos das fezes e de alimento ingerido (Equação 1). A proporção de cada nutriente ingerido que foi excretado nas fezes foi calculada, por rato, a partir das informações dos pesos de sua ingestão e excreção nas fezes (Equação 2). A estimativa para a hidrólise da fração fibra alimentar constituinte das fontes, PRM ou FT, foi calculada, por rato, considerando o seu peso ingerido na fonte fibrosa e o peso determinado nas fezes para o somatório da fibra alimentar e os carboidratos digeríveis, os quais foram resistentes à ação dos sucos gastrintestinais e à degradação pela biota microbiana do intestino grosso (Equação 3).

Equação 1

$$
\text { Total de matéria sólida das fezes }(\%)=\frac{\text { Fezes }}{\text { Alimento ingerido }}
$$

Equação 2

$$
\text { Nutriente excretado nas fezes }(\%)=\frac{\text { Nutriente nas fezes }}{\text { Nutriente ingerido }}
$$

Equação 3

Fibra alimentar hidrolisada (\%) $=\frac{\begin{array}{l}\text { (Fibra alimentar da fonte fibrosa, da ingestão) }- \text { (Fibra } \\ \text { alimentar + Carboidrato digerível, das fezes) }\end{array}}{\text { (Fibra alimentar + Carboidrato digerível, da ingestão) }}$

Os dados foram analisados através da análise de variância e as médias de tratamentos foram comparadas pelo teste de Tukey $(P<0,05)$, segundo Banzatto e Kronka (1995).

\section{Resultados e discussão}

Ratos que receberam as fontes de fibra, polpa refinada de maçã (PRM) ou farelo de trigo (FT), produziram arrastes (Tabela 2$)$ semelhantes $(P>0,05)$, a partir da ingestão para as fezes, tanto do total de matéria sólida quanto dos nutrientes minerais, proteínas, lipídeos e somatório de fibra alimentar da fonte fibrosa mais carboidratos digeríveis da dieta, para todos os níveis testados da fonte de fibra, iguais a $5 \%, 15 \%$ ou $25 \%$ do peso da dieta.

No entanto, acréscimos nas dietas da fonte PRM ou da FT produziram efeitos diferenciados $(P<0,05)$ no arraste (Tabela 2$)$ para as fezes. $\mathrm{O}$ acréscimo de $5 \%$ de PRM não promoveu arraste $(P>0,05)$ para as fezes do total de matéria sólida, de minerais, de proteínas, de lipídeos e do somatório de fibra alimentar da fonte fibrosa mais carboidratos digeríveis da dieta, em comparação com o controle 
(Sem FF). Já, a partir de 15\% de acréscimo da PRM na dieta, com exceção dos lipídeos, houve $(P<0,05)$ arraste para as fezes das demais variáveis. Os lipídeos foram significativamente $(P<0,05)$ arrastados para as fezes apenas quando os ratos receberam dieta contendo 25\% de PRM.

Com relação à fonte FT, apenas os minerais foram significativamente $(P<0,05)$ arrastados para as fezes, já a partir do acréscimo de 5\% desta na dieta. O somatório de fibra alimentar da fonte fibrosa mais os carboidratos digestíveis da dieta e o total de matéria sólida foram significativamente $(P<0,05)$ arrastados com o acréscimo de $15 \%$ de FT, mas, para os nutrientes proteínas e lipídeos o arraste foi significativo $(P<0,05)$ somente quando a dieta recebeu $25 \%$, em peso, da fonte FT (Tabela 2).

Tabela 2 - Proporção (\%) ${ }^{*}$ de fezes secas (total de matéria sólida) e de nutrientes excretados nas fezes em relação ao ingerido pelo rato.

\begin{tabular}{|c|c|c|c|c|c|c|c|c|}
\hline \multirow[t]{2}{*}{ Variável } & \multirow{2}{*}{$\begin{array}{c}0 \% \\
\text { Sem FF }\end{array}$} & \multicolumn{2}{|c|}{$5 \%$} & \multicolumn{2}{|c|}{$15 \%$} & \multicolumn{2}{|c|}{$25 \%$} & \multirow{2}{*}{$\begin{array}{l}\text { Desvio } \\
\text { padrão }\end{array}$} \\
\hline & & PRM & FT & PRM & FT & PRM & FT & \\
\hline $\begin{array}{l}\text { Total de matéria sólida das } \\
\text { fezes }\end{array}$ & $3,20_{c}$ & $5,81_{c}$ & $6,16_{c}$ & $12,72_{\mathrm{ab}}$ & $10,67_{b}$ & $16,12_{a}$ & $14,53_{a}$ & $(1,95)$ \\
\hline Minerais & $13,28_{c}$ & $21,23_{b c}$ & $28,31_{\mathrm{ab}}$ & $29,10_{\mathrm{ab}}$ & $27,42_{\mathrm{ab}}$ & $30,31_{\mathrm{ab}}$ & $30,73_{a}$ & $(5,11)$ \\
\hline Proteínas & $5,88_{d}$ & $8,78_{\mathrm{cd}}$ & $7,72_{\mathrm{cd}}$ & $13,69_{\mathrm{ab}}$ & $9,47_{\mathrm{bcd}}$ & $15,61_{\mathrm{a}}$ & $11,64_{a b c}$ & $(2,44)$ \\
\hline Lipídeos & $1,47_{c}$ & $1,93_{c}$ & $2,61_{\mathrm{bc}}$ & $3,30_{a b c}$ & $3,60_{\mathrm{abc}}$ & $5,43_{a}$ & $4,68_{\mathrm{ab}}$ & $(1,49)$ \\
\hline $\begin{array}{l}\text { Fibra alimentar da fonte } \\
\text { fibrosa e Ch. digerível da } \\
\text { dieta }\end{array}$ & $1,61_{d}$ & $3,78_{d}$ & $3,70_{d}$ & $10,72_{\text {bc }}$ & $9,24_{c}$ & $14,49_{a}$ & $13,23_{\mathrm{ab}}$ & $(1,75)$ \\
\hline
\end{tabular}

*Grupo de seis ratos; Sem FF=sem fonte de fibra; PRM=polpa refinada de maçã, descarte sólido que sobra do processamento da maçã para produção de suco, na presença de enzimas pectinolíticas, seguido de lavagem com água, secagem ao sol e trituração em processador de alimentos; FT=farelo de trigo obtido do comércio; Dado dentro dos parênteses corresponde ao desvio padrão.

A fibra alimentar constituinte da fonte PRM ou FT apresentou grau de hidrólise estimado, respectivamente, em: $48,94 \%$ ou $11,40 \%$ para os grupos que receberam $15 \%$ da fonte de fibra e $59,04 \%$ ou $26,82 \%$ para os grupos que receberam $25 \%$ de fonte de fibra. Para os grupos que receberam $5 \%$ da fonte, apenas a fibra alimentar da PRM foi hidrolisada no mínimo em $45,15 \%$, sendo que para a fibra da fonte FT não foi estimado nenhum grau de hidrólise. Portanto, a fibra alimentar da fonte PRM foi a mais degradada pela biota microbiana do trato digestivo do rato.

A PRM produziu, segundo Raupp et al. (2000), efeitos nas características das fezes e nas defecações dos ratos considerados normais e próprios de uma fibra alimentar. Ainda, seus efeitos nos parâmetros nutricionais, como na ingestão de alimento, no ganho de peso corpóreo, no quociente de eficiência alimentar (QEA) e no quociente de eficiência protéica operacional (PERop) foram assemelhados aos produzidos pelo padrão FT, uma fonte convencional de fibra alimentar para a alimentação humana. Os autores concluiram, naquela pesquisa, que o produto PRM, pela quantidade industrial disponível, por seu conteúdo altíssimo de fibra alimentar igual a $91,91 \%$, peso seco, e principalmente por sua propriedade de funcionalidade digestiva, se constitui em boa fonte alternativa do nutriente fibra alimentar, portanto, numa nova fonte de ingrediente fibroso para a alimentação humana.

A despeito do benefício e do crescente consumo de fibras alimentares na dieta humana, estudos com animais (incluindo os ratos) e com humanos atribuíram a algumas fibras alimentares efeitos adversos específicos, como o de interferir na digestão-absorção de nutrientes conjuntamente ingeridos na dieta (Schweizer 
e Edwards, 1992).

A partir de um outro subproduto gerado pelas fecularias foi produzido, também por Raupp et al. (2002), um produto farináceo chamado de "Bagaço de Mandioca Hidrolisado (BMH)”, o qual apresentou 60,9\% de fibra alimentar, menor proporção que a PRM. Em ensaios biológicos com ratos, o BMH produziu efeito mais pronunciado que o farelo de trigo (FT) em arrastar para as fezes os minerais (globalidade) e a proteína, para as doses de $5 \%$ ou $15 \%$. Na dose alta, de $25 \%$, as fontes de fibra alimentar produziram efeitos semelhantes em arrastar a proteína, mas o FT promoveu maior arraste de minerais (globalidade). Com relação ao efeito sobre o arraste de lipídeos e de carboidratos digeríveis para as fezes dos ratos, não houve dferença entre as fontes BMH e FT.

Os resultados obtidos na pesquisa atual para a PRM, por terem sido assemelhados aos produzidos para o FT, indicaram o seu uso como um ingrediente fibroso para a formulação de alimentos destinados ao consumo humano. Por conseguinte, esse descarte da agroindústria de maçã, por sua composição nutricional e características funcionais digestivas (Raupp et al., 2000), bem como por suas propriedades nutricionais digestivas avaliadas na pesquisa atual, pode se constituir em produto alimentício para ser comercializado como uma farinha de maçã contendo elevado grau de concentração da fibra alimentar insolúvel, diferenciada, portanto, dos produtos fibrosos disponíveis no mercado.

A industrialização desse produto poderá ser viável, também, sob o ponto de vista tecnológico e econômico, mas é recomendável, sobretudo, com relação ao aspecto ambiental. Essa inovação, além de resolver o problema da poluição ambiental, poderá incrementar o rendimento já estabelecido nas indústrias de maçã e colocar à disposição do mercado um novo produto alimentício fibroso potencial.

\section{Agradecimentos}

Os autores agradecem a Novo Nordisk Bioindustrial do Brasil Ltda, Araucária-PR, pela doação das enzimas; à Universidade Federal de Santa Catarina por fornecer os ratos e permitir a realização dos ensaios biológicos.

\section{REFERENNCIAS}

1. AREAS, M.A. Estudo dos efeitos da polpa de laranja sobre parâmetros fisiológicos, nutricionais, bioquímicos e morfológicos em ratos normais e diabéticos. Campinas, 1994. 158p. Tese (Doutorado em Ciência de Alimentos) - Faculdade de Engenharia de Alimentos, Universidade Estadual de Campinas (Unicamp) - Brasil.

2. ASSOCIATION OF OFFICIALANALYTICAL CHEMISTS. Official Methods of Analysis. 14.ed. Washington: AOAC, 1984.

3.BANZATTO, D.A.; KRONKA, S.N. Experimentação Agrícola. 3.ed. Jaboticabal: FUNEP/UNESP-FCAV, 1995. 247p.

4. CARSON, K.J.; COLLINS, J.L.; PENFIELD, M.P. Unrefined, dried apple pomace as a potential food ingredient. Journal of Food Science, v.59, n.6, p.1213-1215, 1994.

5. CHEN, H.; RUBENTHALER, G.L.; LEUNG, H.K.; BARANOWSKI, J.D. Chemical, physical, and baking properties of apple fiber compared with wheat and oat bran. Cereal Chemistry, v.65, n.3, p.244-247, 1988a.

6. CHEN, H.; RUBENTHALER, GL.; SCHANUS, E.G. Effect of apple fiber and cellulose on the physical properties of wheat flour. Journal of Food Science, v.53, n.1, p.304-305, 1988b.

7. DAVIES, J.R.; BROWN J.C.; LIVESEY, G. Energy values and energy balance in rats fed on suplements of guar gum or cellulose. British Journal of Nutrition, v.65, p.415-433, 1991.

8. EASTWOOD, M. A.; BRYDON, W. G.; BAIARD, J. D.; ELTON. R. A; HELLIWELL, S.; SMITH, J. H.; PRITCHARD, J.L. Faecal weight and composition, serum lipids, and diet among subjects aged 18 to 80 years not seeking health care. American Journal of Clinical Nutrition, v.40, p.628-634, 1984.

9. KROTKIEWSKI, M. Effect of guar-gum on body weight, hunger ratings and metabolism in obese subjects. British Journal of Nutrition, v.52, p.97-105, 1984.

10. PROTZEK, E.C.; FREITAS, R.J.S.; WASCZYNSKJ, N. Aproveitamento do bagaço de maçã na elaboração de biscoitos ricos em fibra alimentar. Boletim do Centro de Pesquisa e Processamento de Alimentos, v.16, n.2, p.263-275, 1998.

11. RAUPP, D.S.; CARRIJO, K.C.R.; COSTA, L.L.F.; MENDES, S.D.C.; BANZATTO, D.A. Propriedades funcionais digestivas e nutricionais de polpa refinada de maçã. Scientia Agrícola, v.57, n.3, p.395-402, 2000.

12. RAUPP, D.S.; MARQUES, S.H.P.; ROSA, D.A.; CALDI, C.M.; CREMASCO, A.C.V.; BANZATTO, D.A. Arraste via fecal de nutrientes da ingestão produzido por bagaço de mandioca hidrolisado. Scientia Agrícola, v.59, n.2, p.235-242, 2002.

13. RAUPP, D.S.; SGARBIERI, V.C. Efeitos de frações fibrosas extraídas de feijão (Phaseolus vulgaris, L.) na utilização de macro e micronutrientes da dieta pelo rato. Ciência e 
Tecnologia deAlimentos, v.16, n. 2, p.100-107, 1996.

14. REEVES, P. G.; NIELSEN, F. H.; FAHEY J ., G. C. AIN-93 Purified diets for laboratory rodents: Final report of the American Institute of Nutrition Ad Hoc Writing Committee on the Reformulation of the Ain- $76^{\mathrm{A}}$ Rodent Diet. The Journal of Nutrition, v.123, p.1939-1951, 1993.

15. RENARD, C.M.G.C.; THIBAULT, J.F. Composition and physico-chemical properties of apple fibres from fresh fruits and industrial products. Lebensmittel Wissenschaft und Technologie, v.24, p.523-527, 1991.

16. ROEHRIG, K. The physiological effects of dietary fiber. Food Hydrocolloids, v.2, p.1-18, 1988.

17. SCHNEEMAN, B.O. Soluble vs insoluble fiber: different physiological responses. Food Technology, v.2, p.81-82, 1987.

18. SCHWEIZER, T.F.; EDWARDS, C.A. Dietary Fibre - A Component of Food, Nutritional Function in Health and Disease. London: Ed. Springer-Verlag, 1992. 354p.

19. SEVA-PEREIRA, A.; MORAES, G.R.; OLIVEIRA, S.P.; REYES, F.G.R. Uso de biscoito rico em fibras no tratamento da constipação intestinal crônica. Revista Paulista de Medicina, v.109, p.265-268, 1991.

20. SMITH, A.N.; DRUMMOND.E.; EASTWOOD, M.A. The effects of coarse and fine Canadian Red Spring Wheat and French Soft Wheat bran on colonic motility in patients with diverticular disease. American Journal of Clinical Nutrition, v.34, p.2460-2463, 1981.

21. TOMA, R.B.; CURTIS, D.J. Dietary fiber: effect on mineral bioavailability. Food Technology, v.2, p.111-116, 1986.

22. WALKER. A.R.P. Effect of high crude fiber intake on transit time and the absorption of nutrients in South African Negro schoolchildren. American Journal of Clinical Nutrition, v.28, p.1161-1169, 1975.

23. WALTER, R.H.; RAO, M.A.; SHERMAN, R.M.; COOLEY, H.J. Edible fibers from apple pomace. Journal of Food Science, v.50, n.5, p.747-749, 1985.

24. WANG, H.J.; THOMAS, R.L. Direct use of apple pomace in bakery products. Journal of Food Science, v.54, n.3, p.618$620,1989$. 